\title{
Transfusion Associated Graft Versus Host Disease: A Case Report and Review of Literature
}

\author{
Divya Doval ${ }^{1}$, Sanjeev K. Sharma ${ }^{1}$, Meet Kumar ${ }^{1}$, Vipin Khandelwal ${ }^{1}$, Anil Handoo², Tina Dadu ${ }^{2}$, \\ Dharma Choudhary ${ }^{1}$
}

${ }^{1}$ Department of Hemato-oncology and Bone Marrow Transplantation, BLK Superspeciality Hospital, New Delhi, India

${ }^{2}$ Department of Hematology, BLK Superspeciality Hospital, New Delhi, India

\section{Email address:}

divyadoval@yahoo.co.uk(D.Doval), sksanjeev13@yahoo.com(S.K. Sharma),kr_meet@yahoo.com(M. Kumar),

khandelwal_vipin@yahoo.com(V. Khandelwal), ahhemat@yahoo.co.in (A. Handoo),dadu.tina@gmail.com (T. Dadu),

dharmabmt@gmail.com (D.Choudhary)

\section{To cite this article:}

Divya Doval, Sanjeev K. Sharma, Meet Kumar, Vipin Khandelwal, Anil Handoo, Tina Dadu, Dharma Choudhary. Transfusion Associated Graft Versus Host Disease: A Case Report and Review of Literature. International Journal of Clinical and Experimental Medical Sciences. Vol. 5, No. 3, 2019, pp. 46-48. doi: 10.11648/j.ijcems.20190503.11

Received: May 28, 2019; Accepted: July 17, 2019; Published: August 7, 2019

\begin{abstract}
Transfusion associated graft versus host disease (TA-GvHD) is a rare but usually fatal complication of transfusion of cellular blood products. It is seen usually in immunosuppressed individuals due to engraftment of viable $\mathrm{T}$ lymphocytes but it may also occur in immunocompetent individuals. Patients present with fever, skin rash, diarrhoea, hepatic dysfunction and bone marrow aplasia which manifests as pancytopenia. The diagnosis of TA-GvHD is often delayed because of lack of awareness due to the non-specific manifestations and often these symptoms are attributed to the underlying illness. We present here a case report of fatal TA-GvHD in an immunocompetent patient, post coronary artery bypass grafting surgery after transfusion of blood products. The patient died 27 days after a blood transfusion. An increased risk of TA-GvHD following bypass grafting and other surgical procedures where cardiopulmonary bypass is required has been perceived. TA-GvHD is under reported and the incidence is felt to be too low to warrant routine irradiation of cellular products. Physicians, surgeons and transfusion centers should be aware of this rare but devastating complication of blood transfusion so as to either diagnose it early or rather prevent it.
\end{abstract}

Keywords: Transfusion Associated GvHD (TA-GvHD), Immunocompetent Host, Post CABG

\section{Introduction}

Transfusion associated graft versus host disease (TA-GvHD) is a rare but usually fatal complication of transfusion of cellular blood products. [1, 2] It was first reported in 1955. It is seen usually in immunosuppressed individuals but it may also occur in immunocompetent individuals The diagnosis of TA-GvHD is often delayed because of lack of awareness due to the non-specific manifestations and often these symptoms are attributed to the underlying illness. Unlike GvHD associated with hematopoietic cell transplantation (HCT), TA-GvHD mainly affects the recipient's bone marrow and is almost always fatal.[3] Spontaneous resolution of TA-GvHD [4] and also a few survivors of TA - GVHD have been documented [5]. Also an increased risk of TA-GvHD following bypass grafting where cardiopulmonary bypass is required has been reported. [6]

\section{Case Report}

A 55-year-old diabetic and hypertensive male was admitted with the history of moderate to high grade fever for 10 days associated with chills. He also had a history of a maculopapular rash over his entire body along with generalized dryness of the skin and a loss of appetite and nausea for 3-4 days. He had undergone a coronary artery bypass grafting $(\mathrm{CABG})$ for triple vessel disease 18 days back and was discharged 4 days later. There was no history of bleeding from any site, bony pain and altered bowel or bladder habits. He had received 4 units of fresh packed red blood cells from his relatives during CABG. On admission he had a temperature of $103^{\circ} \mathrm{F}$, pulse rate $120 / \mathrm{min}$, regular, and blood pressure of $100 / 64 \mathrm{~mm} \mathrm{Hg}$. He had a toxic look with mild 
icterus and pallor. He also had a generalized erythematous maculopapular rash over face, trunk and extremities (Figure 1). There was no lymphadenopathy or hepatosplenomegaly. The operated site was healthy.

His hemogram showed hemoglobin $8.4 \mathrm{~g} / \mathrm{dl}$, total leukocyte count $0.45 \times 10^{9} / 1$ and platelet count $73 \times 10^{9} / 1$; differential counts: lymphocytes $87 \%$ and monocytes $13 \%$. Peripheral blood film showed pancytopenia, with absent neutrophils. Reticulocyte count was $0.3 \%$. Liver function test showed total bilirubin $1.98 \mathrm{mg} / \mathrm{dl}$, direct bilirubin $0.97 \mathrm{mg} / \mathrm{dl}$, SGOT/SGPT 200/318 U/1, with normal renal function. Vitamin B12 was $823.6 \mathrm{pg} / \mathrm{ml}$ and serum ferritin was $4220 \mathrm{ng} / \mathrm{ml}$. Blood and urine culture were sterile. Fibrinogen and triglycerides levels were normal. Echocardiogram showed severely hypokinetic mid anterior septum and apex with left ventricular ejection fraction $40 \%$. Bone marrow examination showed hypocellular marrow with presence of agranulocytosis and erythroblastopenia (Figure 2). The patient was treated conservatively with broad spectrum antibiotics as per hospital policy for febrile neutropenia with G-CSF and irradiated blood products. He was continued on supportive care but died of severe sepsis and septic shock 10 days after admission to our center, 27 days after the transfusion.

\section{Discussion}

TA-GVHD results from viable immunocompetent donor lymphocytes attacking recipient's antigen presenting tissues. This manifests clinically by dysfunction of the skin, liver, gastrointestinal tract and bone marrow. The effect on the bone marrow distinguishes TA-GvHD from post transplant acute GvHD. In stem cell transplant, the bone marrow cells in the recipient are of donor origin and are thus spared of the immune attack. Bone marrow aplasia is the primary cause of death in TA-GvHD [7]. Though rare, TA-GVHD has been reported in immunocompetent individuals, [8] who are heterozygous for an HLA haplotype for which the donor is homozygous.[9] TA-GvHD can also develop in patients who are immunosuppressed because of disease (e.g. Hodgkin lymphoma) or because of the chemotherapy (e.g. fludarabine), who receive non-irradiated cellular blood products. An inability of the host's immune system to recognize and/or eliminate foreign donor cells in allogeneic blood components has been found to be the key mechanism in TA-GvHD. [10] Engraftment and expansion of CD4 + and cytotoxic CD8+ donor derived $\mathrm{T}$ cell clones occurs. These cells recognise HLA class II determinants in the recipient, resulting in massive release of inflammatory cytokines such as tumour necrosis factor and interleukin 1 . Subsequently there is recruitment and activation of T cells, upregulation of HLA class II expression and widespread tissue damage. [11]

A systematic review of TA-GvHD found that partial HLA matching was present in $71 \%$ individuals for whom HLA data was available. [10] The incidence of TA-GvHD in immunocompetent recipients is not known, but may be related to genetic variability within a population, as well as the incidence of consanguineous marriage [12]. Any patient receiving any HLA-matched blood components from a blood-related donor should be considered at risk for TA-GvHD. This risk increases in regions with increased genetic homogeneity.[13] The reported frequency of one way matching or sharing HLA haplotype in a non-first degree relative in Japan ranges from 1 in 312 to 1 in 874, and as a result more than 200 cases of TA-GVHD have been reported in immunocompetent individuals in Japan. [14, 15]

Patients developing TA-GvHD present with fever, erythematous maculopapular rash, cough, anorexia, vomiting, abdominal pain and profuse diarrhoea. The main laboratory findings are pancytopenia with a hypocellular marrow, abnormal liver function tests and electrolyte abnormalities. The diagnosis of TA-GvHD is frequently delayed because the symptoms are non specific or are attributed to the underlying illness. Diagnosis is therefore based on clinical and laboratory findings and biopsy results. It can be confused with severe viral illness or adverse reaction in severely ill patients. Therefore the most important step towards the diagnosis of TA-GVHD is to suspect TA-GVHD in patients presenting with non specific manifestations and pancytopenia. The diagnosis is suggested from biopsy of affected skin, which classically reveals vacuolization of the basal layer and a histiocytic infiltrate. [16] The definitive diagnosis can be established only if the circulating lymphocytes are shown to have a different HLA phenotype from host tissue cells proving that they came from the donor. [17]

TA-GvHD in immunocompetent individuals appears commonly following CABG and other cardiovascular surgery in which cardiopulmonary bypass is required. The reasons are not entirely clear but the use of relatively fresh blood which has more viable lymphocytes increases the chances of engraftment [18]. In Japan, the association between TA-GVHD and cardiac surgery has been recognised for some time. This is now attributed to a combination of HLA haplotype sharing and the use of relatively fresh blood. Because such cases are rare outside Japan, irradiated blood is not currently recommended for cardiac surgery patients. [19] The risks and benefits of very fresh blood need to be considered in this context.

There is no effective treatment of TA-GvHD. Corticosteroids, cyclosporine, azathioprine, methotrexate, and stem cell transplant have been used to treat TA-GVHD. Successful treatment with methylprednisolone, cyclosporine in combination with OKT3, and cyclophosphamide, antithymocyte globulin combined with autologous peripheral blood progenitor cell infusion, as well as after allogeneic stem cell transplantation have been reported.[10, 16] There are also reports of spontaneous resolution of TA-GvHD. However, the overall mortality of TA-GvHD is greater than $90 \%$. At present, prevention remains the best strategy.

Our patient did not have any history of previous transfusion of blood or blood products. All the blood products transfused to him during the surgery were non-irradiated and were obtained from his relatives. The patient succumbed to marrow failure following TA-GvHD. Though universal irradiation of blood products before $\mathrm{CABG}$ is not recommended, TA-GvHD 
can be prevented by inactivating transfused lymphocytes by exposing all cellular blood products to gamma irradiation or avoiding directed donations.[19] Leucodepletion of cellular blood products is not a suitable alternative for irradiation.

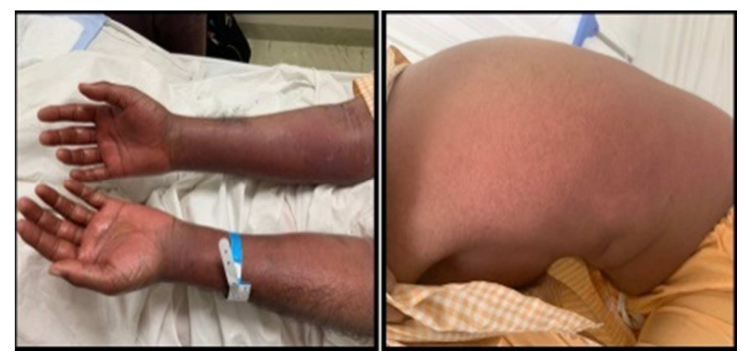

Figure 1. Pictures of arms and back of the patient showing diffuse erythematous maculo-papular rash in the patient with TA-GvHD.

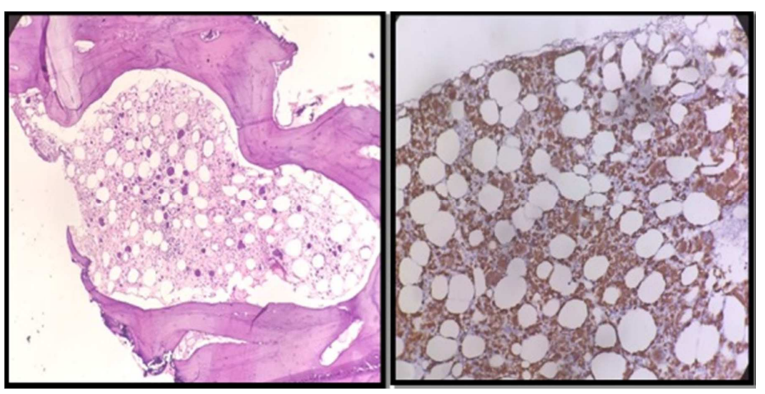

Figure 2. Bone marrow biopsy showing marrow hypoplasia with a marked paucity of granulocytic and erythroid precursors. There is marked prominence of histiocytes as highlighted by CD68 immunostain with some showing evidence of hemophagocytosis.

\section{Conclusion}

TA-GvHD is a rare complication which can manifest as bone marrow failure following blood product transfusion and is almost always fatal. This case highlights the need for a high index of suspicion in any case post $\mathrm{CABG}$ presenting with skin manifestations and pancytopenia.

Compliance with ethical standards.

\section{References}

[1] Williamson LM, Warwick RM,: Transfusion-associated graft-versus-host disease and its prevention. Blood Reviews9, $1995,251-261$.

[2] Brubaker DB: Transfusion-associated graft-versus-host disease Hum Pathol, 1986, 17: 1085-1088.

[3] Nishimura M, Uchida S, Mitsunaga S,: Characterization of T-cell clones derived from peripheral blood lymphocytes of a patient with transfusion-associated graft-versus-host disease: Fas-mediated killing by CD4 + and CD8 + cytotoxic T-cell clones and tumor necrosis factor beta production by CD4 + T-cell clones. Blood, 1997, 89: 1440-1445.
[4] Mori S., Matsushita H., Ozaki K., et al: Spontaneous resolution of transfusion-associated graft-versus-host disease. Transfusion 1995, 35: pp. 431-435.

[5] Hutchinson K., Kopko P. M., Muto K. N., et al: Early diagnosis and successful treatment of a patient with transfusion-associated GvHD with autologous peripheral blood progenitor cell transplantation. Transfusion 2002; 42: pp. $1567-2572$.

[6] Ahyaa R, Douglasb JG, Watsonc HG: Transfusion associated graft versus host disease in an immunocompetent individual following coronary artery bypass grafting. Heart 1998; 80: 299-300.

[7] Ferrara JL, Deeg HJ.: Graft-versus-host disease. N Engl J Med, 1991, 324: 667.

[8] Sakakibara T, Juji T.: Post-transfusion graft-versus-host disease after open heart surgery. Lancet, 1986, 2: 1099.

[9] Shivdasani RA, Haluska FG, Dock NL. Brief report: graft-versus-host disease associated with transfusion of blood from unrelated HLA-homozygous donors. N Engl J Med, 1993328: 766.

[10] Kopolovic I, Ostro J, Tsubota H, et al.: A systematic review of transfusion-associated graft-versus-host disease. Blood, 2015, 126: 406 .

[11] Ferrara J. L., and Reddy P.: Pathophysiology of graft-versus-host disease. Semin Hematol 2006; 43: pp. 3-10.

[12] Aoun E, Shamseddine A, Chehal A.: Transfusion-associated GVHD: 10 years' experience at the American University of Beirut-Medical Center. Transfusion, 2003 43: 1672.

[13] Schroeder ML.: Transfusion-associated graft-versus-host disease. Br J Haematol, 2002117: 275.

[14] Takahashi K, Juji T, Miyazaki H: Post transfusion graft versus host disease occurring in non immunosuppressed patients in Japan. Transfus Sci, 1991, 12: 281-289.

[15] Juji T, Takahashi K, Shibata Y, et al.: Post-transfusion graft versus-host disease in immunocompetent patients after cardiac surgery in Japan. N Engl J Med, 1989, 321: 56.

[16] Dwyre DM, Holland PV. Transfusion-associated graft-versus-host disease, Vox Sanguinis, 2008, 95, 85-93.

[17] Thaler M., Shamiss A., Orgad S., et al: The role of blood from HLA-homozygous donors in fatal transfusion-associated graft-versus-host disease after open-heart surgery. N Engl J Med 1989; 321: pp. 25-28.

[18] Aybey B., Coskun D., and Aytac J.: Transfusion associated graft versus host disease following cardiovascular surgery. Mikrobiyol Bul 2006; 40: pp. 103-107.

[19] Loren D Fast, Preventing transfusion-associated graft-versus-host disease: state of the art International Journal of Clinical Transfusion Medicine 2015: 2. 